\title{
基于地形因子的藏狐生境评价及其空间容纳量的估算
}

\author{
刘群秀 ${ }^{1,2}$ ，王小明 ${ }^{1 *}$ * \\ （1. 华东师范大学 生命科学学院, 上海 200062; 2. 上海动物园, 上海 200335)
}

摘要: 依据藏狐的 “出现点” 数据, 基于藏狐对家域内地形因子的利用, 作者对青海省都兰县沟里乡野生藏 狐的生境质量进行初步评价, 并在基于个体藏狐最小空间需求的基础上, 估算了研究地区的空间环境容纳量。选 择海拔、坡度、坡位和地形起伏度等 4 个主要的地形因子，应用Bonferroni置信区间比较藏狐对各因子的利用及其 可获得性来建立单个因子评价准则, 并依据各因子的分布特征进行综合生境评价。研究结果: 藏狐最适宜的海拔 高度为 $4050 \sim 4300 \mathrm{~m}$ ，坡度为 $5 \sim 20^{\circ}$ ，坡位为上坡位和下坡位; 藏狐对地形起伏度因子没有明显的选择性。 根据藏狐对不同生境因子的利用, 及不同藏狐个体生境利用的一致性, 建立的综合评价准则为: $X i=\left(X_{\text {海拨 }}^{2} \times X_{\text {坡垃 }}^{2_{2}} \times\right.$ $\left.X_{\text {圾度 }} \times X_{\text {地形赽位 }}\right)^{1 / 6}$ 。研究地区内适宜性区域面积约为 $17.4 \mathrm{~km}^{2}$, 占总研究地区面积的 $38.8 \%$, 其中最适宜生境仅 0.4 $\mathrm{km}^{2}$ 。藏狐家域面积从 $2.53 \sim 4.99 \mathrm{~km}^{2}$ 不等, 不同个体间家域重叠指数（OI）从 $0.16 \sim 0.66$ 不等。藏狐的最小空间 需求为 $2.09 \sim 3.55 \mathrm{~km}^{2}$, 研究地区藏狐的空间容纳量为 7 12 只。根据生境资源现状及监测结果表明, 研究地区的 藏狐种群数量保持稳定。

关键词: 藏狐; 生境; 评价准则; 空间容纳量

中图分类号：Q959.838; Q958.11 文献标识码：A 文章编号：0254-5853-(2009)06-0679-08

\section{Implementation of Topography Factors in Habitat Assessment and Spatial Carrying Capacity Estimation for Tibetan Fox}

\author{
LIU Qun-xiu ${ }^{1,2}$, WANG Xiao-ming ${ }^{1, *}$ \\ (1. College of Life Sciences, East China Normal University, Shanghai 200062, China; 2. Shanghai Zoo, Shanghai 200335, China)
}

\begin{abstract}
Based on "Presence data", we assessed the habitat quality according to the physical factor utilized by Tibetan fox in Gouli Township, Dulan County, Qinghai Province, China. The spatial carrying capacity of Tibetan fox was also estimated according to their minimum space requirements. A total of 4 topographical factors, elevation, slope, terrain ruggedness and slope position, were used to conduct habitat assessment for Tibetan fox. The suitability of habitat factors were determined by comparing the utilization by Tibetan fox and its availability based on 95\% Bonferroni confidence interval. A comprehensive assessment criterion was set up for all topographic factors. For Tibetan fox, the elevations of $4050-4300 \mathrm{~m}$, the slopes of $5-20^{\circ}$, the upper and lower slope positions were suitable habitat. Tibetan fox use terrain ruggedness non-selectively. According to the utilization of Tibetan fox on different topographic factors and the consistence of habitat utilization by different fox individuals, we set the comprehensive criterion as: $X i=\left(X_{\text {elevation }}^{2} \times X_{\text {slope position }}^{2} \times\right.$ $\left.X_{\text {slope }} \times X_{\text {terrainrug gadness }}\right)^{1 / 6}$. The area of suitable habitat is about $17.4 \mathrm{~km}^{2}$, which take a percentage of $38.8 \%$ of research area. The area of most suitable habitat is only $0.4 \mathrm{~km}^{2}$. The home range of Tibetan fox ranges from $2.53 \mathrm{~km}^{2}$ to $4.99 \mathrm{~km}^{2}$, and the overlap index (OI) of different individuals were $0.16-0.66$. The minimum space requirements of Tibetan fox are 2.09 $-3.55 \mathrm{~km}^{2}$, and the spatial carrying capacity of Tibetan fox in suitable habitat is $7-12$. According to the habitat resource and monitoring results, we infer that the Tibetan fox population is keeping steady.
\end{abstract}

Key words: Tibetan fox; Habitat; Assessment criterion; Spatial carrying capacity

Hutchinson（1957）把生态位看作是动物生存 条件的总和, 所有环境资源共同构成了物种的多维
超体积生态位。认识野生动物对其生境的利用方式 对于物种的保护和管理至关重要（Boyce＆

收稿日期：2009-07-13; 接受日期：2009-10-14

基金项目：华东师范大学与蒙大纳大学合作项目：青海省藏狐与生境的关系及其它生物学特性的研究; 华东师范大学优秀博士生培养基金

*通讯作者 (Corresponding author), E-mail: xmwang@ecnu.edu.cn

第一作者简介: 男, 博士, 主要从事动物生态学及保护生物学研究 
McDonald, 1999)。把动物活动点与环境变量的信息 相结合建立生境适宜性模型 (Habitat suitability model), 来评价特定物种的生境质量、预测潜在的 适宜生境及物种的地理分布 (Ray \& Burgman, 2006）是决策者在生物多样性保护, 物种监测和管 理中的重要工具和有效手段 (Boyce et al, 2002)。 常用的生境预测模型包括线性回归 (McCullagh \& Nelder，1989）、逻辑斯谛回归（Gavashelishvili \& Lukarevskiy, 2007; Johnson et al, 2006; Keating \& Cherry, 2004)、主成分分析 (Debinski \& Brussard, 1994) 和判别分析 (Livingston et al, 1990) 等。众 多生境预测模型为野生动物种群的保护及其生境 的科学管理提供重要的信息, 在保护生物学和野生 动物研究中得到快速发展 (Klar et al, 2008) 和广泛 应用（Jin et al, 2008; Hunter, 1996)。

我们以青海省都兰县沟里乡藏狐 (Vulpes ferrilata) 为研究对象, 应用 “出现点” (Presence) 数据建立生境评价模型, 基于地形因子的分布特征 进行其生境评价, 同时依据适宜生境的面积和藏狐 个体的最小空间需求估算空间环境容纳量。我们选 择 “出现点” 数据有以下两个原因: (1) 藏狐活动 较快, 不容易被发现, 很难确定其不利用的区域, 在数据处理中不能简单地将 “未记录” 等同于 “不 利用”; (2) 藏狐对某些区域的 “不利用” 可能是 一种暂时的, 受天气或人类活动影响的 “假性不利 用” (false absences) (Hirzel et al, 2006), 调查过程 中可能获得错误信息。通过比较不同藏狐个体的生 境选择特征确定生境因子对该物种的重要性, 并探 讨不同个体间生境喜好的共同特征。在研究地区内 确定物种对生境的利用, 将有助于我们: (1) 设计 和改善监测和取样方法, 进一步分析物种对环境变 化的适应性策略; (2) 有助于确定对目标物种具有 保护价值的区域, 以将其纳入优先保护计划中, 从 而为野生藏狐种群的科学保护和管理提供理论指 导。

\section{1 研究地区}

研究地区位于青海省都兰县沟里乡 (都兰国际 狩猎场内), 该区地处青藏高原北部山地向高原主 体过渡地段的东昆仑山支脉布尔汗布达山 $\left(\mathrm{N} 35^{\circ}\right.$ $34^{\prime}$, E $98^{\circ} 36^{\prime}$ ), 海拔 $3900 \sim 5000 \mathrm{~m}$, 面积约 $45 \mathrm{~km}^{2}$ 。 研究地区属高原高寒大陆性气候, 干旱多风, 昼夜 温差大, 蒸发强烈, 年平均蒸发量 $2021 \mathrm{~mm}$, 无霜
期短（约 $157 \mathrm{~d}$ ), 年平均温度 $3.8^{\circ} \mathrm{C}$ (Bai, 2005)。 降水多集中在 6 至 9 月, 约占全年降水的 $80 \% \sim 90$ $\%$ (Zhou et al, 1987)。研究地区地势开阔, 地形起 伏不平, 植被类型单一, 主要为高山草原和高山草 甸, 以金露梅 (Potentilla fruticosa) 为主的灌从和 牧草 (Jiang \& Xu, 2006)。阳坡、半阳坡草本植物 生长良好, 灌丛主要生长在沟谷和阴坡, 呈零星分 布。每年 5 月植被开始返青, 9 月进入枯黄期。研 究地区分布的优势植物有紫花针茅 (Stipa purpurea)、猪毛菜 (Salsola collina)、早熟禾 (Poa alpina) 和沙蒿 (Artemisia desertorum) 等。野生动 物资源主要包括盘羊 (Ovis ammon)、岩羊 (Pseudois nayaur)、藏原羚 (Procapra picticaudata)、狼 (Canis lupus)、赤狐 (Vulpes vulpes)、喜马拉雅旱獭

(Marmota himalayana)、高原鼠兔 (Ochotona curzoniae) 和中华鼠鼠 (Myospalax fantanieri) 等 （Liu et al, 2007; Bai, 2005; Zhou et al, 1987)。在 研究地区藏狐的潜在天敌包括家犬、狼（Canis lupus)、金雕 (Aquila chrisaetos) 等 (Wang et al, 2008 ), 其中家犬数量较多, 分布广泛。

\section{2 研究方法}

我们在研究地区共捕捉 4 只野生藏狐（3 雄 1 雌), 佩戴无线电项圈 ( Telonics Co. Ltd. USA, Mesa, Arizona, MOD-225, 重量 $120 \mathrm{~g}$ ) 后释放, 并于 2006 年 9-10月， 2007 年 3-5 月和 9-10 月， 2008 年 3-4 月采用 Homing 定位法进行野外无线电遥测 (Mech, 1983; Liu et al, 2007), 并记录藏狐活动位 点。遥测频率为 1 次/ (只 $-\mathrm{d})$, 遥测时间为 7:0018: 00 , 为避免遥测时间对实验数据产生影响, 对 每一只藏狐, 从 7: 00 点开始遥测, 每天推后 $1 \mathrm{~h}$, 至 18: 00 截止, 不同个体交替进行遥测。研究期 间我们共记录藏狐活动位点 422 个, 其中雄 1(fox1, m) 134 个; 雌 1（fox2, f) 138 个; 雄 2（fox3, m) 119 个; 雄 3 (fox4, m) 31 个。

\section{1 生境因子的提取}

基于研究地区等高线图（1：100000, 海拔间 隔 $20 \mathrm{~m}$ ), 应用 ArcView GIS 3.3 a (ERSI Inc, 2002) 制作数字化高程模型 (digital elevation model, DEM) $(90 \mathrm{~m} \times 90 \mathrm{~m})$ 。研究中应用的地形因子主要 包括: (1) 海拔 (elevation, 单位: $m$ ): 利用 ArcView GIS 空间分析扩展模块（spatial analysis extension） 从 DEM 中提取; (2) 坡度 (slope, 单位: ${ }^{\circ}$ ): 从 
DEM 中提取, 方法同 (1); (3) 地形起伏度 (terrain ruggedness): 采用ArcView GIS 扩展模块 Areas and Ratios from Elevation Grid, v1.2 计算地形表面与其 垂直投影平面面积的比例 (比值越大, 说明地势起 伏越明显)（Jenness，2002); (4) 坡位（slope position): 应用 ArcView GIS 扩展模块 Topographic position index 计算 (Jenness, 2006), 将坡位划分为: 山顶、上坡位、中坡位、下坡位，山谷和沟底等 6 大类。

\section{2 评价准则的建立}

生境选择的研究常通过比较动物对生境的利 用和生境可获得性来确定动物主动选择的环境类 型（Thomas \& Taylor, 2006; Jansen et al, 2000)。 Dettmers \& Bart (1999)提出当动物对某种生境类型 的利用最大程度地高于环境因子可获得性时, 此生 境类型可以定义为该物种的适宜生境。本研究中, 我们假设藏狐能够到达研究地区所有生境范围, 参 照 Neu et al（1974）对动物生境利用的比较方法, 计算 Bonferroni 置信区间（95\%）来比较藏狐对地 形因子不同属性范围的利用和该因子的可获得性 进行因子适宜性评价并赋值（图 1)。根据不同藏狐 个体对地形因子利用的比较结果, 将藏狐生境评价 标准划分为最适宜 $(4-5)$ 、适宜 $(3-4)$ 、次适宜 (2-3) 和不适宜 (1-2) 等 4 大类 (图 1)。3 作 为适宜生境 (Good habitat) 与非适宜生境 (Others) 的分界点。

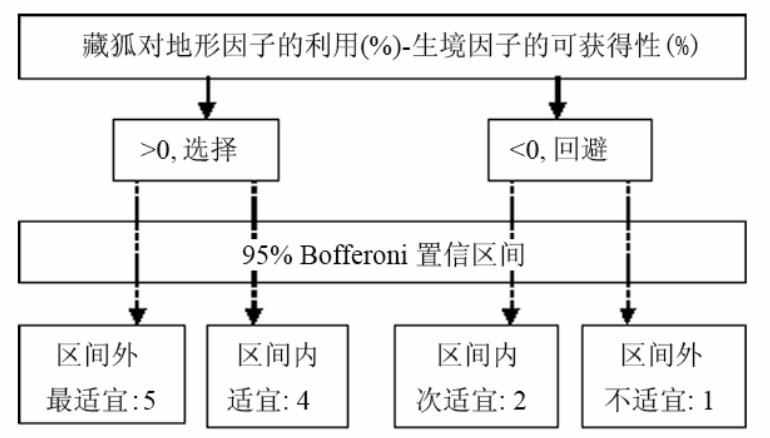

图 1 因子评价准则的建立

Fig. 1 Illustration on the assessing of habitat factors

\section{3 空间模拟与分析}

我们以评价准则为基础, 分析海拔、坡度、地 形起伏度和坡位等单一生境因子的适宜性特征, 然 后根据生态位适宜度模型, 应用 ArcGIS 9.2 将各地 形因子进行综合分析 (Tang \& Yang, 2006), 确定沟 里乡藏狐总体生境适应性的分布特征。各因子综合
计算公式如下:

$$
X_{j}=\left(\prod_{i=1}^{n} X_{i j}\right)^{1 / n}
$$

其中, $X_{j}$ 为生态位适宜度指数, $X_{i j}$ 为第 $i$ 个 生境因子第 $j$ 类级别的生态位适宜度指数（Ouyang et al, 1996)。

\section{4 空间容纳量估算}

应用 ArcGIS 9.2 的 Hawth's Tools 工具计算藏狐 家域（95\%固定核空间法）（ESRI，2002），参照 Atwood \& Weeks (2003)计算不同藏狐个体间家域 的重叠指数 (overlap index, OI), 公式如下:

$$
O I=\left[\left(\text { area }_{\alpha \beta} / h r_{\alpha}\right)\left(\text { area }_{\alpha \beta} / h r_{\beta}\right)\right]^{0.5}
$$

其中, area $_{\alpha \beta}$ 为 $\alpha$ 和 $\beta$ 个体家域重叠部分的 面积, $h r_{\alpha}$ 和 $h r_{\beta}$ 分别为 $\alpha$ 和 $\beta$ 个体的家域面积。

根据不同藏狐个体家域及重叠, 计算藏狐的最 小空间需求 (minimum space requirements, MSR):

$$
M S R=h r_{i} \times\left(1-\bar{x}_{O I} / 2\right)
$$

其中, $h r_{i}$ 为藏狐个体 $i$ 的家域面积, $\bar{x}_{O I}$ 为该 藏狐个体与其他个体家域重叠指数的平均值。

参照 Downs et al（2008），我们应用适宜生境 面积来估算容纳量, 并根据藏狐家域中非适宜生境 的百分比对研究地区适宜生境面积进行调整, 计算 公式为:

$K=\frac{\text { 适宜生境总面积 } \times(1+\text { 藏狐家域中非适宜生境的百分比 })}{\text { 藏狐个休的最小空间雷求 }}$

\section{3 结 果}

\section{1 藏狐对地形因子的利用及综合评价准则的建} 立

藏狐的活动主要集中在海拔 $4050 \sim 4300 \mathrm{~m}$, 并对 4 150 4 $250 \mathrm{~m}$ 的区域表现出明显的选择性 (HSI $>4$ ), 而对 $4000 \mathrm{~m}$ 以下及 $4300 \mathrm{~m}$ 以上的区 域则表现为回避（图 2)，不同个体的选择利用方式 比较一致（表 1); 藏狐对坡位因子的选择主要集中 在上坡位和下坡位（HSI $>4 ）$, 而对中坡位明显回避 $(\mathrm{HSI}<2)$, 且不同个体呈现高度一致; 藏狐对缓坡 度区域 (5 20 $\left.0^{\circ}\right)$ 呈现正选择, 而回避较陡的坡度 
区域 $\left(30 \sim 40^{\circ}\right)$; 地形起伏度对藏狐活动无显著影

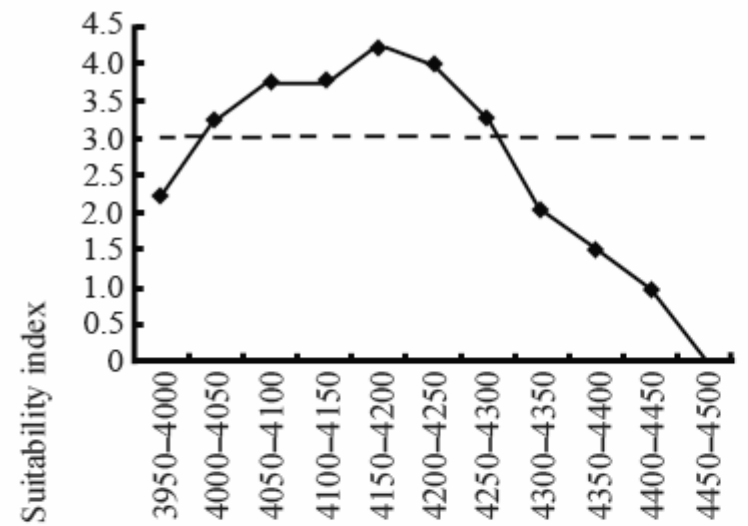

海拔 Elevation
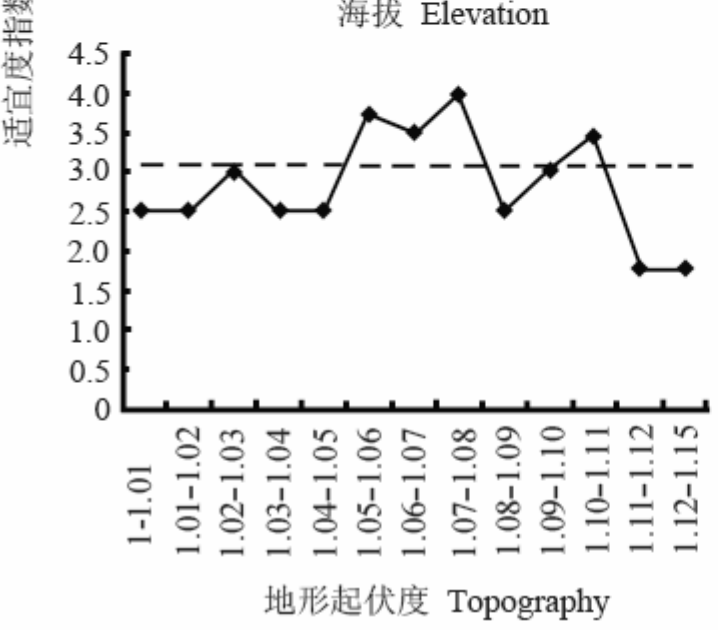

响，且不同个体间选择性不一致（图 2, 表 1)。

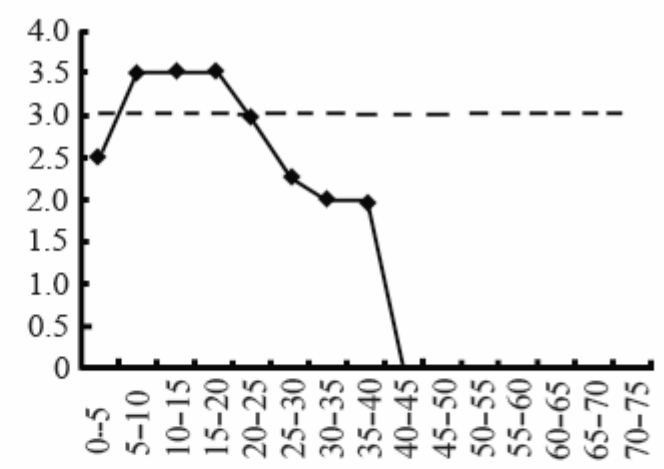

坡度 Slope

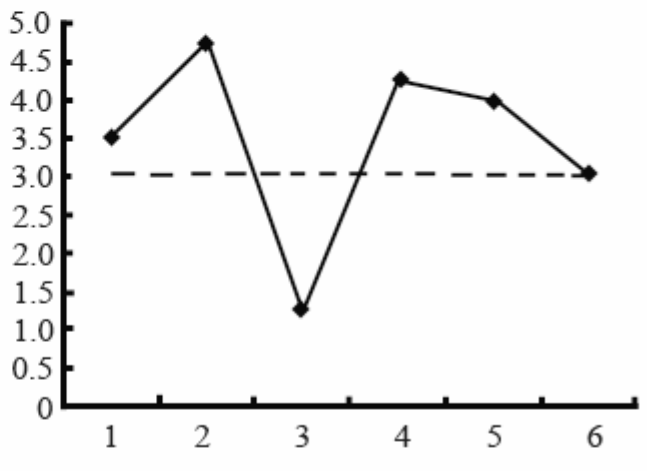

坡位 Slope position

图 2 藏狐对生境因子的选择

Fig. 2 Habitat selection of Tibetan fox in Gouli Township, Dulan County, Qinghai Province

表 1 不同藏狐个体对地形因子利用的相关性分析

Tab. 1 Correlation of terrain factor attributes utilized by different Tibetan fox individuals

\begin{tabular}{lcc}
\hline 因子类别 Terrain factors & 生境利用相关性 $(\%){ }^{*}$ Correlation between individuals & 因子利用的显著选择 Significant selections \\
\hline 海拔 Elevation & 83.3 & $\mathrm{y}$ \\
坡位 Slope position & 100.0 & $\mathrm{y}$ \\
坡度 Slope & 100.0 & $\mathrm{n}$ \\
地形起伏度 Terrain ruggedness & 50.0 & $\mathrm{n}$ \\
\hline
\end{tabular}

*不同藏狐个体对因子利用呈显著相关的百分比 (Pearson correlation, 两两检验)。

${ }^{*}$ The percentage of significantly correlated factor utilization patterns of Tibetan foxes (Pearson correlation, for each 2 individuals).

由于藏狐对海拔和坡位因子表现出明显的选 择或回避行为, 且不同藏狐个体的利用方式比较一 致，因此在应用所有地形因子综合评价藏狐生境 时, 我们应用平方项 (Quadratic term) 以突出海拔 和坡位因子对于生境质量的决定作用（Downs et al, 2008 ), 将各地形因子的综合计算公式定义为: $X_{i=}$ $\left(X_{\text {海找 }}^{2} \times X_{\text {圾位 }}^{2} \times X_{\text {坡度 }} \times X_{\text {地形匙优度 }}\right)^{1 / 6}$ 。该公式基于藏狐自身 的生境利用特征, 能够反应不同地形因子在藏狐生
态学需求中的重要作用, 并准确地分析研究地区的 生境特征。

\section{2 生境特征分析}

根据地形因子的综合评价结果, 研究地区的藏 狐的适宜生境面积为 $17.4 \mathrm{~km}^{2}$, 占研究地区总面积 的 $38.8 \%$, 其中最适宜生境面积仅为 $0.4 \mathrm{~km}^{2}$ $(0.9 \%)$, 大部分生境不适宜藏狐活动 $(61.2 \%)$ 。 藏狐的适宜生境主要集中在海拔较低, 靠近河流两 
岸的区域，最适宜生境破碎化程度较高（图 3)。

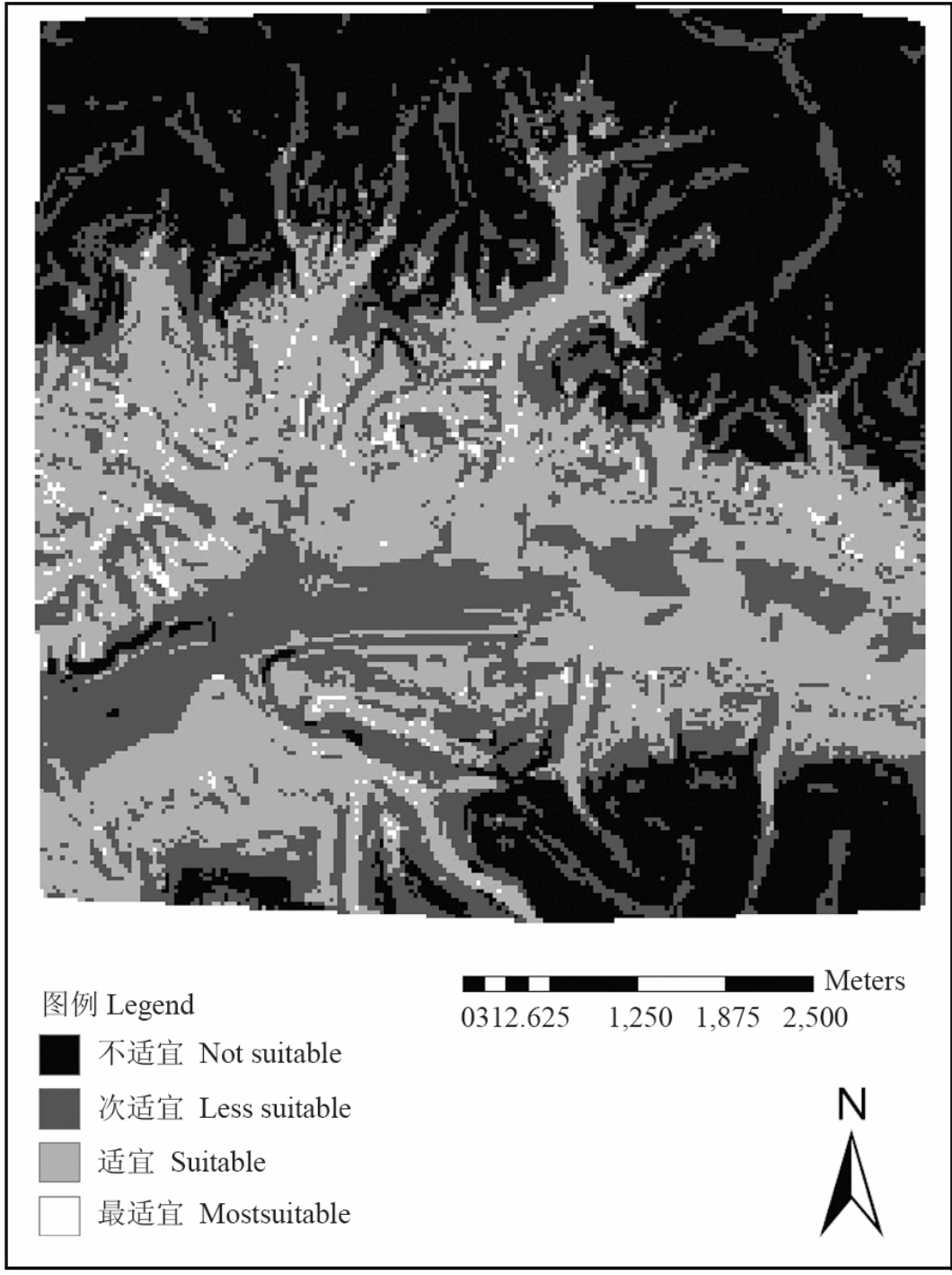

图 3 藏狐家域适宜性评价

Fig. 3 Assessment on the habitat suitability of Tibetan fox

表 2 藏狐个体间家域重叠面积及重叠指数重叠指数 Overlap index

Tab. 2 The overlap area and overlap index of different Tibetan fox individuals

\begin{tabular}{|c|c|c|c|c|c|}
\hline \multirow{2}{*}{\multicolumn{2}{|c|}{$\begin{array}{c}\text { 重叠区面积 } \\
\text { Overlap area }\left(\mathrm{km}^{2}\right)\end{array}$}} & \multicolumn{4}{|c|}{ 重叠指数 Overlap index } \\
\hline & & Fox $13.72 *$ & Fox2 3.48 & Fox3 2.53 & Fox4 4.99 \\
\hline Fox1 & $(63.2 \#)$ & & 0.23 & 0.26 & 0.16 \\
\hline Fox2 & $(66.9)$ & 0.82 & & 0.32 & 0.66 \\
\hline Fox3 & $(70.0)$ & 0.81 & 0.96 & - & 0.24 \\
\hline Fox4 & $(67.6)$ & 0.71 & 2.75 & 0.87 & - \\
\hline
\end{tabular}

*家域面积（Home range area） $\left(\mathrm{km}^{2}\right)$, \#家域中适宜生境百分比（Percentage of suitable habitat in home range）(\%)。

\section{3 空间环境容纳量估算}

4 只藏狐的家域面积从 $2.53 \sim 4.99 \mathrm{~km}^{2}$ $(\bar{x}=3.68, S D=1.01)($ 表 2)，并且藏狐家域彼此 均有重叠, 其重叠指数 $(O I)$ 从 $0.16 \sim 0.66(\bar{x}=0.31$, 
$S D=0.18)$ 。根据藏狐的家域面积及重叠状况, 依据 公式 (3), 我们计算得出藏狐的最小空间需求为 $2.09 \sim 3.55 \mathrm{~km}^{2}$ 。藏狐家域中不适宜生境的比例为 $30 \% \sim 36.8 \% （ \bar{x}=33.1 \% ， S D=2.8$ ）（表 2)，依据藏 狐家域内适宜生境的面积及藏狐的最小空间需求, 则整个研究地区的藏狐空间容纳量为 7 12 只。

\section{4 讨 论}

动物的外部生境可以分为生物和非生物环境 两大类 (Ouyang et al, 2001)。Willson（1974）认为 除了植被类型和组成外, 非生物环境变量能够提高 野生动物生境描述的准确性。已有研究证实, 地表 覆盖物、坡度和坡位等环境因子是藏狐微生境选择 的重要的决定因子 (Wang et al, 2007; Gong \& Hu, 2003 )。选择地形因子的另一个重要原因是生境位 置、土壤质地等对高原鼠兔的密度和分布的影响 (Wang et al, 2004), 在研究地区, 藏狐主要捕食高 原鼠兔 (Liu et al, 2009), 地形因子的结构组成可能 间接地影响藏狐对生境因子的选择利用（Messier， 1985)。本研究未能开展对整个研究地区的高原鼠 兔密度分布的调查, 因此选择地形因子进行藏狐的 生境评价至关重要。地形因子具有比较稳定、容易 辨认等优点 (Ma et al, 2006), 加之对微生境的决定 作用, 选用地形因子进行藏狐的生态学研究能够客 观、真实地反应其生活史需求和生境选择特征。

由于藏狐家域内海拔因子的属性范围 3950 $4450 \mathrm{~m}$, 坡度范围是 $0 \sim 45^{\circ}$, 因此不能确定海拔 高于 $4450 \mathrm{~m}$, 坡度大于 $45^{\circ}$ 的地形区域的适宜性。 Gong \& Hu（2003）认为藏狐活动的坡度范围主要 集中在 $20 \sim 30^{\circ}$, 相对海拔集中在 $100 \sim 200 \mathrm{~m}$, 一 般不选择 $200 \mathrm{~m}$ 以上的区域。本研究中, 藏狐对低 海拔 ( $4000 \sim 4300 \mathrm{~m})$ 、缓坡度 $\left(5 \sim 20^{\circ}\right)$ 呈正选 择特点, 因此, 我们认为藏狐不会到海拔大于 4 $450 \mathrm{~m}$ 、坡度大于 $45^{\circ}$ 的区域活动, 基于藏狐家域 内地形因子能够客观、全面地反应藏狐的生物学习 性及其生境质量。本研究是基于地形因子进行藏狐 生境评价, 因此, 研究地区山脉走向及分布是生境 适宜性的重要影响因子。研究地区地形起伏不平

(Jiang \& Xu, 2006), 平均海拔为 $4336.5 \mathrm{~m}$ $(S D=209.2 \mathrm{~m})$, 适宜的海拔区域 (4050 4300 m) 主要分布于河流的两岸, 并深入分布于山脉之间

(图 3)。该部分区域对于藏狐的适宜性可能源于低 海拔处丰富的高原鼠兔资源（Wang et al, 2004）和
水源。同其他研究相比, 本研究中适宜生境面积百 分比较小, 并且部分区域被分隔成条状 (图 3)。藏 狐行动敏捷（Feng et al, 1986), 可以快速通过障碍 物寻找适宜生境, 形成了适应青藏高原恶劣环境的 适应性生存策略 (Gong \& Hu, 2003)。藏狐经常在 缓坡处捕食猎物, 但不会远离可以逃避天敌的陡峭 地形。尽管研究地区适宜生境面积较小 $(38.8 \%)$, 但对于藏狐这一物种, 不适宜生境可能被其利用行 使不同的生物学功能, 因此地形因子对藏狐活动规 律的影响有待于更深入的探讨和研究。

人类活动是造成野生动物生境丧失和种群下 降的主要原因之一 (Xu et al, 2006; Meffe \& Carroll, 1994)。近年来, 人类活动的加剧使青藏高原的草 地数量及质量均持续下降 ( Wu \& Du, 2007)。在研 究地区影响藏狐分布的人类活动主要包括以下两 个方面: (1) Wang (2005) 提出人类活动的干扰和 过度放牧对草场的破坏直接威胁着藏狐的生存。研 究地区放牧季节 (每年的 5-10月) 牛、羊 (500 1000 只) 的大面积活动会对藏狐的活动产生直接影 响, 另外牧群的过度啃食会使草场退化, 使生境质 量变低, 从而影响藏狐的活动; (2) 在研究地区, 鼠兔被认为是破坏草场畜牧承载量的有害物种而 遭到毒杀（Lai \& Smith, 2003; Xu et al, 2002)。作 为藏狐的重要食物组成, 鼠兔数量的急剧减少会破 坏原有的生态平衡, 从而影响藏狐的生境选择与利 用。因此, 在资源管理过程中, 建立重点保护区域 是寻求野生动物保护与人类用地活动之间的平衡 点的重要条件, 对于物种与生境的长远保护至关重 要 (Kideghesh, 2006)。

动物的家域面积可能受种群密度等诸多因素 的影响 (Hubbs \& Boonstra, 1998; Wollf, 1985), 种 群密度的升高会引起动物家域面积的减小或家域 重叠程度的增大 (Erlinge et al, 1990; Priotto \& Steinmann, 1999), 藏狐的最小空间需求可能随之发 生变化。研究期间, 所有遥测藏狐的家域面积都比 较稳定, 并呈现领域行为, 我们在研究地区适宜生 境范围共计数到 8 只藏狐, 此调查结果与容纳量的 估算结果基本一致。猎物资源的丰富度可能是食肉 动物分布的重要影响因子 (Messier, 1985), 在环境 物理因子适宜的前提下，高原鼠兔的密度分布可能 会成为藏狐最小空间需求的决定因素。研究地区高 原鼠兔资源丰富 (Liu, 2005), 藏狐的最小空间需求 可能随外来干扰、竞争等存在弹性变动。 
致谢：本研究得到 Robert M. Lee 基金会和 Denver 动物学基金会的资助, 国际野生生物保护学 会 (WCS) 的 RFP 项目资助, 在此表示感谢。野 参考文献:

Atwood TC, Weeks HP. 2003. Spatial home range overlap and temporal interaction in eastern coyotes: the influence of pair types and fragmentation [J]. Can J Zool, 81(9): 1589-1597.

Bai SL. 2005. Investigation and classification on native grassland resources in Dulan County [J]. Qinghai Prataculture, 14(3), 51-55.[白生立. 2005. 都兰县天然草地资源调查报告. 青海草业, 14(3): 51-55.]

Boyce M, McDonald L. 1999. Relating populations to habitats using resource selection function [J]. Trends Ecol Evol, 14(7): 268-272.

Boyce MS, Vernier PR, Nielsen SE, Schmiegelow FKA. 2002. Evaluating resource selection functions [J]. Ecol Model, 157(2-3): 281-300.

Debinski DM, Brussard PF. 1994. Using biodiversity data to assess species-habitat relationships in Glacier National Park, Montana [J]. Ecol Appl, 4(4): 833-843.

Dettmers R, Bart J. 1999. A GIS modeling method applied to predicting forest Songbird habitat [J]. Ecol Appl, 9(1): 152-163.

Downs JA, Gates RJ, Murray AT. 2008. Estimating carrying capacity for sandhill cranes using habitat suitability and spatial optimization models [J]. Ecol Model, 214: 284-292.

Erlinge S, Hoogenboom I, Agrell J, Nelson J, Sandell M. 1990. Density-related home-range size and overlap in adult field voles (Microtus agrestis) in southern Swede [J]. J Mammal, 71(4): 597-603.

ESRI (Environmental Systems Research, Inc.). 2002. ArcView 3.3 [M]. California, USA: ESRI, Redlands

ESRI Inc. (2006) ESRI ArcMAP? 9.2 ed.(M)

Feng ZJ, Cai GQ, Zheng CL. 1986. Mammals in Tibet [M]. Beijing: Science Press. [冯祚建, 蔡桂全, 郑昌琳. 1986. 西藏哺乳类. 北京: 科学出 版社.]

Gavashelishvili A, Lukarevskiy V. 2007. Modelling the habitat requirements of leopard Panthera pardus in west and central Asia [J]. J App Ecol, 45(2): 579-588.

Gong MH, Hu JC. 2003. The Summer Microhabitat Selection of Tibetan Fox in the Northwest Plateau of Sichuan [J]. Acta Theriol Sin, 23(3): 266-269. [龚明昊, 胡锦青. 2003. 四川西北高原藏狐的夏季微生境 选择. 兽类学报, 23(3): 266-269.]

Hirzel AH, Lay GL, Helfer V, Radin C, Guisan A. 2006. Evaluating the ability of habitat suitability models to predict species presence [J]. Ecol Model, 199(2): 142-152.

Hubbs AH, Boonstra R. 1998. Effects of food and predators on the home range size of arctic ground squirrels (Spermophilus parryii) [J]. Can $J$ Zool, 76: 592-596.

Hunter ML. 1996. Fundamentals of conservation biology [M]. Blackwell Science, Cambridge, Massachusetts, USA.

Hutchinson GE. 1957. Concluding remarks [J]. Cold Spring Harb Symp Quant Biol, 22: 415-427.

Jansen R, Little RM, Crowe TM. 2000. Habitat utilization and home range of the redwing francolin, Francolinus levaillantii, in highland grasslads, Mpumalanga province, South Africa [J]. 38(4): 329-338.

Jenness J. 2002. Surface areas and ratios from elevation grid (surfgrids.avx) extension for Arcview 3.x, v.1.2. Jenness enterprises[EB/OL]. http://www.jennessent.com/arcview/surfacesareas.htm.

Jenness J. 2006. Topographic Position Index (tpi_jen.avx) extension for ArcView 3.x, v. 1.3a. Jenness Enterprises[EB/OL]. http://www.jennessent.com/arcview/tpi.htm.

Jiang ZG, Xu AC. 2006. Snow Leopold [J]. Chn J Zool, 41(4): 128. [蒋志刚, 徐爱春. 2006. 雪豹. 动物学杂志, 41(4): 128.]
外工作中, 都兰国际狩猎场场长达盛林, 助手周家 克给予大力支持和帮助, 在此一并表示感谢。

Jin LR, Sun KP, He HS, Zhou YF. 2008. Research advances in habitat suitability index model [J]. Chn J Ecol, 27(5): 841-846. [金龙如，孙克 萍, 贺红士, 周宇飞. 2008. 生境适宜度指数模型研究进展. 生态学 杂志, 27(5): 841-846.]

Johnson CJ, Nielsen SE, Merrill EH, McDonald TL, Boyce MS. 2006. Resource selection functions based on use-availability data: theoretical motivation and evaluation methods [J]. $J$ Wildl Manage, 70(2): 347-357.

Keating KA, Cherry S. 2004. Use and interpretation of logistic regression in habitat-selection studies [J]. J Wildl Manage, 68(4): 774-789.

Kideghesh JR. 2006. Wildlife conservation and local land use conflicts in western Serengeti Tanzania [D]. PhD Dissertation, Trondheim: Norwegian University of Science and Technology.

Klar N, Fernández N, Kramer-Schadt S, Herrmann M, Trinzen M, Büttner I, Niemitz C. 2008. Habitat selection models for European wildcat conservation [J]. Biol Consevr, 141(1): 308-319.

Lai CH, Smith AT. 2003. Keystone status of plateau pikas (Ochotona curzoniae): effect of control on biodiversity of native birds [J]. Biodiver Conserv, 12: 1901-1912.

Liu DS. 2005. The sustainable development of livestock in Dulan County [J]. Qinghai J Anim Vet Sci, 35(1): 23-24. [刘东升. 2005. 浅谈都兰县 畜牧业的可持续发展. 青海畜牧兽医杂志, 35(1): 23-24.]

Liu QX, Harris RB, Wang XM. 2009. Food habits of Tibetan fox (Vulpes ferrilata) in the Kunlun Mountains, Qinghai Province, China [J]. Mammal Biol. (in press).

Liu QX, Harris RB, Wang XM, Wang ZH. 2007. Home range size and overlap of Tibetan foxes Vulpes ferrilata in Dulan County, Qinghai Province [J]. Acta Theriol Sin, 27(4): 370-375. [刘群秀, 王小明, 王正 寰. 2007. 青海省都兰县沟里乡藏狐家域及重叠度. 兽类学报, 27(4): 370-375.]

Livingston SA, Todd CS, Krohn WB, Owen RB. 1990. Habitat model for nesting Bald Eagles in Maine, USA [J]. J Wildl Manage, 54(4): 644-653.

Ma MD, Luo CJ, Zhang J, Hu TX, Liu YJ. 2006. Quantitative classification of site condition in natural forest of Picea asperata [J]. Chn J Eco-Agric, 14(2): 159-163. [马明东, 罗承德, 张 健, 胡庭兴, 刘跃 建. 2006. 云杉天然林分生境条件数量分类研究. 中国生态农业学 报, 14(2): 159-163.]

McCullagh P, Nelder JA. 1989. Generalized linear models (Second edition). Monographs on statistics and applied Probability Number 37 [M]. Chapman and Hall. London, UK.

Meffe GK, Carroll R. 1994. Principle of conservation biology [M]. Sinauer Associates, Sunderland.

Messier F. 1985. Social organization, spatial distribution, and population density of wolves in relation to moose intensity [J]. Can J Zool, 63 : 1068-1077.

Neu CW, Byers CR, Peek JM. 1974. A technique for analysis of utilization-availability data [J]. J Wildl Manage, 38(3): 541-545.

Ouyang ZY, Liu JG, Xiao H, Tan YC, Zhang HM. 2001. An assessment of giant panda habitat in Wolong nature reserve [J]. Acta Ecol Sin, 21(11): 1869-874. [欧阳志云, 刘建国, 肖 寒, 谭迎春, 张和民. 2001. 卧 龙自然保护区大熊猫生境评价. 生态学报, 21(11): 1869-874.]

Ouyang ZY, Wang RS, Fu GN. 1996. Ecological niche suitability and its application in land suitability assessment [J]. Acta Ecol Sin, 16(2): 113-120. [欧阳志云, 王如松, 符贵南. 1996. 生态位适宜度模型及 
其在土地利用适宜性评价中的应用. 生态学报, 16(2): 113-120.]

Priotto JW, Steinmann AR. 1999. Factors affecting home range size and overlap in Akodon azarae (Muridae: Sigmodontinae) in natural pasture of Argentina [J]. Acta Theriol Sin, 44: 37-44.

Ray N, Burgman MA. 2006. Subjective uncertainties in habitat suitability maps [J]. Ecol Model, 195(3-4): 172-186.

Tang GA, Yang X. 2006. Spatial analysis of ArcGIS in Geographic information system [M]. Beijing: Science Press. [汤国安, 杨 昕. 2006. ArcGIS 地理信息系统空间分析实验教程. 北京: 科学出版社.]

Thomas DL, Taylor EJ. 2006. Study designs and tests for comparing resource use and availability II [J]. J Wildl Manage, 70(2): 324-336.

Wang ZH. 2005. The ecology of Tibetan fox (Vulpes ferrilata) in Shiqu County, Sichuan Province, China [D]. Shanghai: PhD dissertation of East China Normal University.[王正嬛. 2005. 四川省石渠县藏狐的 生态学研究. 上海: 华东师范大学博士学位论文.]

Wang ZH, Wang XM, Lu QB. 2007. Selection of land cover by the Tibetan fox Vulpes ferrilata on the eastern Tibetan Plateau, western Sichuan Province, China [J]. Acta Theriol, 52(2): 215-223.

Wang ZH, Wang XM, Chmura AA. 2008. Den habitat characteristics of Tibetan foxes (Vulpes ferrilata) in Shiqu County, Sichuan Province, China [J]. Zool Stud, 47(4): 445-454.

Wang Y, Wang XM, Wang ZH, Giraudoux P, Kenichi Takahashi, Alastair G. 2004. Primary Study on Habitat Choice of Plateau Pika (Ochotona curzoniae) [J]. J Sichuan Univ; Natural Science Edition, 41(4): 1041-1045. [王淯, 王小明, 王正寰, Giraudoux P, Kenichi
Takahashi, Alastair G. 2004. 高原鼠兔生境选择的初步研究. 四川大 学学报(自然科学版), 41(4): 1041-1045.]

Willson MF. 1974. Avain community organization and habitat structure [J]. Ecology, 55(5): 1017-1029.

Wollf JO. 1985. The effects of density, food, and interspecific interference on home range size in Peromyscus leucopus and Peromyscus maniculatus [J]. Can J Zool, 63: 2657-2662.

Wu GL, Du GZ. 2007. Discussion on Ecological Construction and Sustainable Development of Degraded Alpine Grassland Ecosystem of the Qinghai-Tibetan Plateau [J]. Chn J Nat, 29(3): 159-164. [武高林, 杜国祯. 2007. 青藏高原退化高寒草地生态系统恢复和可持续发展 探讨. 自然杂志, 29(3): 159-164.]

Xu SX, Zhao XQ, Sun P, Zhao W, Zhao TB. 2002. The Situation of the Grassland Rodent Harms and Measures of Elimination in the Qing hai Province [J]. Acta Ecol Anim Dom, 23(1): 47-49. [徐世晓, 赵新全, 孙 平, 赵 伟, 赵同标. 2002. 青海省草地鼠害现状及其治理. 家畜生 态学报, 23(1): 47-49.]

Xu WH, Ouyang ZY, Li Y, Liu JG. 2006. Habitat assessment on giant panda in Qinling mountain range based on RS and GIS [J]. Remote Sen Technol Appl, 21(3): 238-242.[徐卫华, 欧阳志云, 李 宇, 刘建国. 2006. 基于遥感和 GIS 的秦岭山系大熊猫生境评价. 遥感技术与应 用, 21(3): 238-242.]

Zhou XM, Wang ZB, Du Q. 1986. Vegetation in Qinghai [M]. Xining: Qinghai People Press. [周兴民, 王质杪, 杜 庆. 1986. 青海植被. 西宁: 人民出版社.]

\section{华东师范大学生命科学学院生态学教研室简介}

华东师范大学生命科学学院生态学教研室主要研究方向包括种群生物学和生态学。目前从事动物生态 学的研究和教学工作, 主要研究领域涉及濒危动物的保护生物学、种群生物学、分子生态学等。负责科研 项目十余项，受到诸如美国野生动物保护协会（WCS）、英国国际动植物区系学会（FFI）、美国国立卫生 研究院（NIH）等国际组织、国家自然科学基金、教育部、上海"曙光计划"、上海市科委、上海第二届引 进海外高层次留学人员基金和上海市重点学科等基金的资助。目前课题组已在国内外学术刊物上发表论文 多篇。 